Michalik, Kerstin

\title{
Empirische Forschung zu Wirkungen des Philosophierens mit Kindern auf die Entwicklung von Kindern, Lehrkräften und Unterricht \\ Philosophieren mit Kindern - Forschungszugänge und -perspektiven. Opladen; Berlin; Toronto : Verlag Barbara Budrich 2018, S. 13-32
}

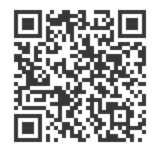

Quellenangabe/ Reference:

Michalik, Kerstin: Empirische Forschung zu Wirkungen des Philosophierens mit Kindern auf die Entwicklung von Kindern, Lehrkräften und Unterricht - In: Philosophieren mit Kindern -

Forschungszugänge und -perspektiven. Opladen; Berlin; Toronto : Verlag Barbara Budrich 2018, S. 13-32 - URN: urn:nbn:de:0111-pedocs-207722 - DOI: 10.25656/01:20772

\section{https://nbn-resolving.org/urn:nbn:de:0111-pedocs-207722}

https://doi.org/10.25656/01:20772

in Kooperation mit / in cooperation with:

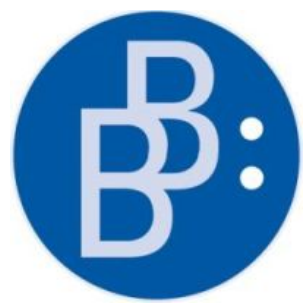

https://www.budrich.de

\section{Nutzungsbedingungen}

Dieses Dokument steht unter folgender Creative Commons-Lizenz: http://creativecommons.org/licenses/by-sa/4.0/deed.de - Sie dürfen das Werk bzw. den Inhalt vervielfältigen, verbreiten und öffentlich zugänglich machen sowie Abwandlungen und Bearbeitungen des Werkes bzw. Inhaltes anfertigen, solange sie den Namen des Autors/Rechteinhabers in der von inm festgelegten Weise nennen und die daraufhin neu entstandenen Werke bzw. Inhalte nur unter Verwendung von Lizenzbedingungen weitergeben, die mit denen dieses Lizenzvertrags identisch, vergleichbar oder kompatibel sind.

Mit der Verwendung dieses Dokuments erkennen Sie die Nutzungsbedingungen an.

\section{Terms of use}

This document is published under following Creative Commons-License: http://creativecommons.org/licenses/by-sa/4.0/deed.en - You may copy, distribute and transmit, adapt or exhibit the work or its contents in public and alter, transform, or change this work as long as you attribute the work in the manner specified by the author or licensor. New resulting works or contents must be distributed pursuant to this license or an identical or comparable license.

By using this particular document, you accept the above-stated conditions of use.

\section{Kontakt / Contact:}

\section{peDOCS}

DIPF | Leibniz-Institut für Bildungsforschung und Bildungsinformation

Informationszentrum (IZ) Bildung

E-Mail: pedocs@dipf.de

Internet: www.pedocs.de

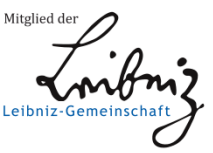


Kerstin Michalik

Empirische Forschung zu Wirkungen des

Philosophierens mit Kindern auf die Entwicklung von

Kindern, Lehrkräften und Unterricht

Verlag Barbara Budrich

Opladen • Berlin • Toronto 2018 
Der Aufsatz Empirische Forschung zu Wirkungen des Philosophierens mit Kindern auf die Entwicklung von Kindern, Lehrkräften und Unterricht von Kerstin Michalik steht unter der Creative Commons Lizenz Attribution-ShareAlike 4.0 International (CC BY-SA 4.0): https://creativecommons.org/licenses/by-sa/4.0/

Diese Lizenz erlaubt die Verbreitung, Speicherung, Vervielfältigung und Bearbeitung bei Verwendung der gleichen CC-BY-SA 4.0-Lizenz und unter Angabe der UrheberInnen, Rechte, Änderungen und verwendeten Lizenz.

Der Aufsatz ist erschienen in:

Michalik, Kerstin/de Boer, Heike (Hrsg.) (2018): Philosophieren mit Kindern - Forschungszugänge und -perspektiven. Opladen: Verlag Barbara Budrich, S. 13-32.

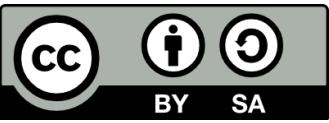

Dieser Beitrag steht im Open-Access-Bereich der Verlagsseite zum kostenlosen Download bereit (https://doi.org/10.3224/84742105.02).

$\begin{array}{ll}\text { ISBN } & 978-3-8474-2105-4 \\ \text { DOI } & 10.3224 / 84742105.02\end{array}$




\section{Empirische Forschung zu Wirkungen des Philosophierens mit Kindern auf die Entwicklung von Kindern, Lehrkräften und Unterricht}

\section{Kerstin Michalik}

Philosophische Gespräche sind seit den 70er-Jahren des 20. Jahrhunderts Gegenstand empirischer Forschung, in deren Rahmen Wirkungen des Philosophierens auf die kognitive, sprachliche, soziale und emotionale Entwicklung von Kindern und Jugendlichen erfasst werden. Der Beitrag gibt einen systematischen Überblick über den aktuellen Stand der empirischen Forschung, die sich vor allem auf den anglo-amerikanischen Sprachraum (Großbritannien, USA, Kanada, Australien) konzentriert.

\section{Einleitung}

Das Philosophieren mit Kindern hat seit den 70er-Jahren weltweite Verbreitung gefunden. Seitdem sind zahlreiche empirische Forschungsarbeiten entstanden, die deutliche Hinweise auf positive Auswirkungen regelmäßiger philosophischer Gespräche auf die Entwicklung von Kindern in ganz unterschiedlichen Bereichen geben. Solche empirischen Befunde sind interessant und wichtig, sie liefern überzeugende Argumente für eine Integration philosophischer Gespräche in Erziehung und Unterricht, auch wenn das Philosophieren in seinen komplexen Dimensionen nicht in jeder Hinsicht erfass- und messbar ist und es unabhängig vom Vorhandensein solcher empirischer Daten für die Kultivierung des philosophischen Gesprächs eine Reihe gewichtiger pädagogischer und schulpolitischer Gründe gibt wie z. B. Kommunikations- und Partizipationsfähigkeiten als übergreifende Bildungsziele.

Empirische Forschung zum Philosophieren mit Kindern gibt es im deutschsprachigen Raum bislang noch wenig, was zum einen daran liegt, dass in der deutschen Philosophiedidaktik empirische Forschung erst in den letzten Jahren zum Diskussionsgegenstand und Arbeitsgebiet geworden ist (vgl. Tiedemann 2004/2011; Rohbeck 2011). Zum anderen ist das Philosophieren mit Kindern in der allgemeinen Erziehungswissenschaft ein noch relativ wenig verbreitetes Arbeitsgebiet. Die Wirkungsforschung zum Philosophieren mit Kindern stammt daher vor allem aus dem anglo-amerikanischen Sprachraum.

In den USA, in England, Kanada und Australien gibt es eine längere Forschungstradition, die bereits in den 70er-Jahren mit Lipmans Gründung des „Institut for the Advancement of Philosophy for Children“(P4C) einsetzte. Die 
überwiegend an Lipmans kinderphilosophischem Ansatz orientierte Forschung konzentrierte sich lange Zeit auf Aspekte der kognitiven Entwicklung: kritisches, logisches, kreatives Denken (,Thinking Skills') und Argumentationsund Gesprächsfähigkeiten, die für Lipmans „Community of Inquiry“, die Forschungsgemeinschaft der philosophierenden Kinder, zentral ist. In den überwiegend mit quantitativen Forschungsmethoden in Gruppen von 8- bis 12-jährigen Kindern durchgeführten Untersuchungen konnten deutliche Effekte im Hinblick auf die kognitive und sprachliche Entwicklung festgestellt werden, wobei jedoch Langzeiteffekte kaum untersucht wurden und die verschiedenen Studien sich methodisch durch ein breites Spektrum auszeichnen und nicht immer den gängigen Standards und Gütekriterien quantitativer Forschung entsprechen (Trickey/Topping 2004; vgl. im Überblick auch Sternberg/Bhana 1996; Cebas/Moriyón o.J. sowie Garcia-Moriyón/Rebollo/Colom 2004). In deutlich geringerem Umfang wurden im Rahmen qualitativer Untersuchungen (Videoanalysen, Interviews) auch Wirkungen im nicht kognitiven Bereich, im Hinblick auf das Sozial- und Gruppenverhalten, auf die soziale und emotionale Entwicklung der Kinder und auf die Entwicklung von Selbstwertgefühl und Selbstvertrauen erforscht (vgl. Fields 2006; Sasseville 1995). In den letzten Jahren hat sich die Wirkungsforschung weiter ausdifferenziert. Neben breit angelegten vor allem quantitativen Untersuchungen im Sinne der frühen Forschungstradition, die Wirkungen regelmäßiger philosophischer Gespräche auf die kognitive und sprachliche Entwicklung von Kinder und Jugendlichen in den Blick nehmen ${ }^{1}$, gibt es eine Reihe von qualitativen und quantitativen Studien, die sich auf nicht-kognitive Aspekte konzentrieren. Hierzu gehören auch Forschung zu Wirkungen des Philosophierens auf soziale und politische Einstellungen und Haltungen von Kindern sowie der relative neue und wichtige Bereich der Forschung zu Wirkungen des Philosophierens auf das Lernen im Fachunterricht und auf Interaktionsmuster im Unterricht. In diesem Forschungszweig steht der Ansatz des Philosophierens als Unterrichtsprinzip im Mittelpunkt und es werden Veränderungen im schulischen Lernen und im professionellen Selbstverständnis von Lehrkräften untersucht.

\section{Wirkungen philosophischer Gespräche auf die kognitive Entwicklung von Kindern und Jugendlichen}

Die neuere Forschung zum Einfluss philosophischen Fragens und Nachdenkens auf verschiedene Aspekte der kognitiven Entwicklung wurde maßgeblich

\footnotetext{
1 Die meisten Studien aus dem anglophonen Bereich basieren auf dem von Lipman entwickelten P4C-Programm oder ähnlichen Ansätzen, d.h. es wird mit verschiedenen Impulsmaterialien und Fragen der Kinder im Sinne der „Community of Philosophical Inquiry“ gearbeitet.
} 
beeinflusst durch die Befunde einer Begleitstudie der University of Dundee zu einem schottischen Schulreformprojekt. Um das Denken der Kinder zu fördern, wurde 2004 das Philosophieren in regulären Grundschulklassen mit 10bis 12-jährigen Kindern in einem ganzen Schuldistrikt in Clackmannanshire flächendeckend für den Zeitraum von 16 Monaten eingeführt und mit standardisierten Testverfahren im Pre-Post-/Test- und Kontrollgruppen-Design untersucht (Topping/Trickey 2007a).

Deutliche Hinweise auf positive Wirkungen wöchentlichen Philosophierens, bei dem die Beschäftigung mit mehrdeutigen, kognitiv herausfordernden Geschichten und Sachverhalten im Mittelpunkt stand, wurden in verschiedenen Bereichen festgestellt: Im Hinblick auf die kognitiven Fähigkeiten der Schüler/innen im Alter von 10-12 Jahren konnten signifikante Verbesserungen in allen verbalen und non-verbalen Bereichen logischen und problemlösenden Denkens festgestellt und die gemessene Intelligenz (um durchschnittlich 6 IQPunkte) verbessert werden. Der höchste Zuwachs zeigte sich bei Schüler/innen im unteren Leistungsbereich (und tendenziell auch bei Jungen), was sich dahingehend deuten lässt, dass das Philosophieren insbesondere für Kinder aus sozial benachteiligten Elternhäusern, deren Sprachfähigkeiten weniger entwickelt sind, förderlich ist (Topping/Trickey 2007a: 285). Eine signifikante Verbesserung konnte auch im Bereich des auf das eigene Lernen bezogenen Selbstwertgefühls der Kinder, die Selbstwahrnehmung als Lernende und aktive Problemlöser im Sinne meta-kognitiven Denkens festgestellt werden (vgl. Topping/Trickey 2007b: 279f.).

$\mathrm{Zu}$ den untersuchten Wirkungen gehörten neben der kognitiven Entwicklung (inklusive metakognitiven Fähigkeiten) auch das Sozialverhalten der Kinder und die Gesprächskultur in der Klasse, die mit qualitativen Verfahren (Videografien, Interviews von Lehrkräften und Schüler/innen) analysiert wurden. Hier wurde eine Verbesserung des Gesprächsverhaltens, der aktiven Beteiligung der Kinder und der argumentativen Fähigkeiten deutlich sowie ein verändertes Frageverhalten der Lehrkräfte hin zu mehr ergebnisoffenen und kognitiv herausfordernden Fragen (vgl. Topping/Trickey 2007b).

Im Rahmen der Untersuchung von Langzeiteffekten zeigte sich in einer zwei Jahre später erfolgten Follow-up-Studie, dass der signifikante Zugewinn an kognitiven Fähigkeiten auch nach dem Übergang in die Sekundarstufe erhalten blieb, obwohl das Philosophieren nicht fortgeführt worden war. Bei den getesteten Schüler/innen aus den Vergleichsgruppen war hingegen eine Verschlechterung der Testergebnisse zu konstatieren, d.h. das Niveau der kognitiven Leistungen hatte nach dem Übergang in die Sekundarstufe gegenüber der Grundschulzeit abgenommen (vgl. Topping/Trickey 2007c).

Die Ergebnisse der schottischen Studie konnten wenige Jahre später (2010/11) durch eine analog angelegte US-amerikanische Untersuchung in texanischen Schulklassen mit 12- bis 13-jährigen Kindern sowohl hinsichtlich der gemessenen Effekte (Fair u.a. 2015a: 31f.) als auch der Langzeitwirkung 
im Rahmen einer nach drei Jahren durchgeführten Folgestudie repliziert werden (Fair u.a. 2015b). Auch in dieser Studie waren die stärksten Effekte bei den sozial benachteiligten Schüler/innen zu verzeichnen, deren Anteil in den texanischen Untersuchungsgruppen jedoch mit rund 60\% deutlich höher lag als in Schottland. Und obwohl der Untersuchungszeitraum mit nur 22-26 Wochen hier deutlich geringer war als in der schottischen Studie, konnten vergleichbar nachhaltige Effekte erzeugt werden.

Eine dezidiert als Langzeitstudie angelegte Untersuchung wird in der Europäischen Schule Madrid durchgeführt, die das Philosophieren mit Kindern und Jugendlichen bereits Mitte der 90er-Jahre des 20. Jahrhunderts in allen Klassenstufen in das Curriculum der Schule integriert hat. Für die Untersuchung werden rund 450 Schüler/innen überwiegend aus der Mittel- und Oberschicht über einen Zeitraum von zehn Jahren begleitet; die Datenerhebung erfolgt zu drei Zeitpunkten, jeweils im Alter von 8 Jahren, 11-12 Jahren und 16 Jahren. Auf der Basis standardisierter Testverfahren werden neben allgemeinen kognitiven Fähigkeiten auch grundlegende Persönlichkeitsmerkmale sowie sprachliche und mathematische Leistungen erhoben (vgl. Colom/Moriyón/Magro/Morilla 2014). Für die ersten zwei Messungszeitpunkte liegen erste Ergebnisse vor, die einen deutlichen Vorsprung der Testgruppen in allen Bereichen zeigen (bei der Intelligenzmessung liegt dieser bei 7 IQ-Punkten). Wie in der schottischen und texanischen Studie sind auch hier die Effekte am deutlichsten bei den Schüler/innen aus den unteren Leistungsbereichen. Ein interessantes Ergebnis ist, dass die Werte aus der Testung zur persönlichen Entwicklung die Kinder aus den Philosophieklassen als pro-sozialer, gleichzeitig aber als emotional etwas instabiler ausweisen, was von der Forschergruppe auf die Besonderheiten eines philosophischen, fragenden und zweifelnden Zugangs zur Welt zurückgeführt wird (ebd.: 55).

Eine weitere Großstudie zu kognitiven Wirkungen des Philosophierens mit Grundschulkindern wurde 2013 in Großbritannien von der „Education Endowment Foundation“, einer gemeinnützigen Stiftung mit dem Ziel, den Zusammenhang zwischen Familieneinkommen und schulischem Erfolg zu reduzieren, in Auftrag gegeben und von der Durham University evaluiert. Untersucht wurden die Auswirkungen eines knapp einjährigen Philosophier-Programms für Grundschüler/innen im Alter von 10-12 Jahren. Neben Testungen zur allgemeinen kognitiven Entwicklung wurden erstmals auch mathematische und Lesekompetenzen untersucht. In diesen Bereichen wurden die größten Effekte erzielt, obwohl weder Lesefähigkeiten noch Mathematik in einem direkten Zusammenhang mit dem Philosophieren stehen. Auch in dieser englischen Untersuchung war die Entwicklung bei Kindern aus sozial benachteiligten Schichten am deutlichsten, was für die Messung der allgemeinen kognitiven Kompetenzen allerdings nicht so deutlich ausgeprägt war (vgl. Gorard/Siddiqui/See 2015: 32). 
Eine wichtige Ergänzung zu den Befunden quantitativer Studien zu Wirkungen regelmäßigen Philosophierens auf die kognitive Entwicklung von Kindern und Jugendlichen stellen die umfangreichen qualitativen Forschungsarbeiten der kanadischen Forscher/innengruppe um Marie-France Daniel dar. Seit mehr als 15 Jahren wird hier u.a. im Rahmen internationaler Forschungskooperationen die kognitive und epistemologische Entwicklung von Kindern im Rahmen philosophischer Gespräche in Kindergarten und Grundschule untersucht. In Erweiterung des quantitativen Paradigmas wurden hier mit qualitativen Forschungsmethoden und Auswertungsverfahren (u.a. Grounded Theory) Interaktionen und Dialoge von Kindern in Kindergruppen und im Fachunterricht sowie Gesprächstranskripte analysiert. Der Schwerpunkt der Forschung lag auf der Untersuchung der Entwicklung dialogisch-kritischen Denkens. In einer ersten Forschungsphase wurden philosophische Gespräche mit Kindern (1012 Jahre) im Mathematikunterricht untersucht, um auf der Basis von Transkript Analysen die Entwicklung verschiedener Formen dialogisch-kritischen Denkens (logisches Denken, kreatives Denken, verantwortungsvolles Denken, metakognitives Denken) im Verlauf eines Jahres zu untersuchen (vgl. Daniel et al. 2002, 2005a, 2005b; Daniel 2008).

Die kanadischen Studien kommen zu dem Ergebnis, dass die soziale Interaktion im Diskurs unter Gleichaltrigen, wie sie für die philosophische „Community of Inquiry“ kennzeichnend ist, wesentlich zur Stimulierung von Denkprozessen höherer Ordnung beiträgt. Es konnte gezeigt werden, dass die Gesprächsbeiträge der Kinder im Untersuchungszeitraum eines Schuljahres deutlich an Umfang, Komplexität und Differenziertheit zunahmen (Daniel 2008: 38ff.). Das in diesem Forschungsrahmen entwickelte Stufenmodell dialogisch-kritischen Denkens unterscheidet fünf Dialogtypen, die vom anekdotischen oder monologischen, d.h. auf das Erzählen eigener Erlebnisse beschränkten Sprechen, über den Austausch von Meinungen bis hin zu einem offenen, verschiedene Standpunkte und Meinungen kritisch überprüfenden und an der Bearbeitung einer gemeinsamen Frage orientierten Dialogs reichen (Daniel et al. 2002; 2005: 338-341).

Im Hinblick auf die Erforschung des Entwicklungsprozesses kritischen Denkens kamen die Untersuchungen zu dem Ergebnis, dass es eine Verbindung gibt zwischen der Entwicklung der Gesprächskultur oder Qualität des Gespräches in der Gruppe und der Entwicklung des individuellen kritischen Denkvermögens der Kinder. Mit der Etablierung eines wirklich „dialogischen“ Gesprächs in den Gruppen im Verlauf des Untersuchungszeitraumes, in dem andere Perspektiven und Meinungen aufgegriffen, diskutiert, weitergedacht und auch in das eigene Denken aufgenommen wurden, gestaltete sich auch das Denken der Kinder zunehmend komplexer und multimodal im Sinne logischen, kreativen, verantwortlichen und meta-kognitiven Denkens (vgl. Daniel u.a. 2005a, 2005b; Daniel u.a. 2002; Daniel u.a. 2011; Daniel 2008). 
In einem zweiten Schritt wurden die in diesem Forschungskontext außerdem deutlich werdenden Korrelationen in der Entwicklung des kritisch-dialogischen Denkens mit der persönlichen epistemologischen Entwicklung der Kinder weiter untersucht im Sinne einer ,relationalen Epistemologie“, die die Entwicklung des Denkens in einen Prozess sozialer Konstruktion situiert, in einen dynamischen Prozess der Konstruktion von Sprache und Denken im Austausch mit anderen. Als Ergebnis wurden drei epistemologische Perspektiven identifiziert: die egozentrische, die relativistische und die intersubjektive Perspektive. Diese Perspektiven erfassen Entwicklungen im Ausmaß und in der Form der Bezogenheit auf die Dialogpartner/innen und stellen gleichzeitig verschiedene Repräsentationen von Welt in den Vorstellungen der Kinder dar (Daniel 2008: 334). In Folgestudien konnte nachgewiesen werden, dass philosophische Gespräche sowohl bei Kindern im Grundschulalter als auch bereits bei sehr jungen Kindern im Alter von vier Jahren zur Entwicklung komplexerer epistemologischer Perspektiven beizutragen vermögen (Daniel/Delsol 2005; Daniel/Gagnon 2012; Daniel/Gagnon/Auriac-Slusarczyk 2017).

Mit diesen auf der Basis qualitativer Analysen in langjährigen Forschungsprozessen gewonnen Erkenntnisse werden die Ergebnisse quantitativer Forschung zu Wirkungen des Philosophierens auf die kognitive Entwicklung von Kindern und Jugendlichen gestützt. Darüber hinaus tragen die Ergebnisse zur Vertiefung und Erweiterung des Verständnisses jener Prozesse bei, die für die Mobilisation und Entwicklung dialogischen-kritischen Denkens maßgeblich sind (Daniel/Gagnon/Auriac-Slusarczyk 2017: 241). In diese Richtung geht auch die explorative Studie Helzels zu kindlichen Entwicklungsprozessen beim Philosophieren, die auf der Basis der dokumentarischen Methode die dialogisch-argumentative Entwicklung von mehrperspektivischem Denken und Strategien zum Umgang mit Ambiguität in einer ersten Grundschulklasse im Verlauf eines Schuljahres exemplarisch untersucht (siehe hierzu den Beitrag von Gudrun Helzel in diesem Band sowie Helzel 2018; Helzel/Michalik 2015). Die Bedeutung interaktiver Kommunikationsprozesse für die Entwicklung komplexerer Denkformen wird auch in der qualitativen Studie Hausbergs zur Genese kreativer Denkweisen oder in den Untersuchungen von de Boer zu Lernprozessen in Gesprächen (2015) deutlich.

\section{Wirkungen philosophischer Gespräche auf die Sprachentwicklung}

In engem Zusammenhang mit den Befunden zu Wirkungen des Philosophierens auf die kognitive Entwicklung von Kindern und Jugendlichen stehen die Ergebnisse zur Entwicklung von Kommunikations- und Sprachfähigkeiten im Rahmen regelmäßigen Philosophierens. Beim Philosophieren steht das 
Gespräch im Mittelpunkt des Geschehens und Sprache und Denken sind eng miteinander verknüpft. In den bereits vorgestellten quantitativen Studien sind teilweise auf der Basis begleitender qualitativer Erhebungen wie Videoaufnahmen von Unterrichtsgesprächen Veränderungen im Gesprächsverhalten (Begründen von Meinungen) und hinsichtlich der Gesprächsanteile von Kinder und Jugendlichen sowie im Frageverhalten von Lehrkräften deutlich geworden (vgl. Topping/Trickey 2007a: 285/2007b: 79ff.). Positive Auswirkungen auf die sprachlich-argumentativen Kompetenzen der Kinder und auf die Gesprächsdynamik zwischen Kindern und Lehrkräften im Sinn einer deutlichen Zunahme der an Mitschüler/innen gerichteten Gesprächsbeiträge hat auch die Forschergruppe um Daniel in ihren verschiedenen qualitativen Analysen philosophischer Gespräche in Kindergarten und Grundschule festgestellt (vgl. Daniel/Delsol 2005: 23f.; Daniel 2008: 38ff.)

Hinweise auf den Einfluss des Philosophierens auf das Kommunikationsund Sprachverhalten gibt es in verschiedenen Studien (vgl. auch Niklasson/Ohlsson/Ringborg 1996), bislang liegt aber nur eine begrenzte Zahl von Spezialuntersuchungen vor. In einer kleinen Studie an einer kanadischen Schule mit einem sehr hohen Anteil von Kindern mit Migrationshintergrund konnten Schleifer und Courtemanche in einer Gruppe von 10-12jährige Kindern mit besonderen Sprachschwierigkeiten im Rahmen eines Pre-Post-Designs mit Test- und Kontrollgruppe eine deutliche Verbesserung des sprachlichen Ausdrucks bei den Kindern, die an einem Philosophieprogramm teilgenommen hatten, feststellen. Diese Verbesserung zeigte sich u.a. im verstärkten Gebrauch von Konnektoren (z.B.: weil, damit, wo), was die Autor/innen der Studie unter anderem auf die besondere Bedeutung logischen und folgerichtigen Denkens im philosophischen Gespräch und auf das Interesse der Kinder, eigene Standpunkte klar und verständlich für andere Gesprächsteilnehmer/innen auszudrücken, zurückführen (Schleifer/Courtemanche 1996: 31). Sprachbildende Effekte des Philosophierens konnten auch in Lerngruppen mit 911jährigen lernbehinderten Kindern (vgl. Simon 1979) und bei Kindern, bei denen die Unterrichtssprache die Zweitsprache war (vgl. Siddiqui/Gorard/See 2017: 28), registriert werden. Beim Philosophieren in einer Fremdsprache zeigten sich ähnliche Effekte, so im Rahmen einer iranischen Studie unter College-Studierenden, bei der in englischer Sprache philosophiert wurde (vgl. Gholamhossein/Siamak 2010). Die Autoren/innen dieser Studien sprechen von einer ,indirekten Form“ des Zweitspracherwerbs, deren sprachfördernde Wirkung unter anderem vermutlich durch die hohe intrinsische Motivation der Teilnehmer/innen, sich in den philosophischen Gesprächen mitzuteilen und eigene Gedanken verständlich zu machen, bedingt sei (ebd.: 720).

Sprachbildende Effekte bei deutschsprachige Kindern sind erstmals im Rahmen einer Studie zur Sprachentwicklung von Kindern im Vorschulalter im Rahmen eines Pre-Post-Untersuchungsdesigns mit einer Test- und Kontrollgruppe auf der Basis von Sprachstandsauswertungen und einer 
kategoriengeleiteten Analyse von Gesprächstranskripten untersucht worden. Hier konnte im direkten Vergleich nachgewiesen werden, dass das Sprechen über komplexe Inhalte in philosophischen Gesprächen die Entwicklung komplexerer Sprachstrukturen bei den Kindern in ungleich größerem Maße als vergleichbare, auf dialogische Interaktionen ausgerichtete Gesprächsmethoden, anregt (siehe den Beitrag von Katrin Alt hierzu im vorliegenden Band).

\section{Wirkung des Philosophierens auf die soziale, emotionale und persönliche Entwicklung}

Philosophische Gespräche, in denen eigene Vorstellungen und Meinungen eingebracht, Standpunkte ausgetauscht und geprüft und gemeinsam an einer offenen Frage oder einem Problem gearbeitet wird, haben auch Auswirkungen auf das Sozialverhalten von Kindern und Jugendlichen und auf ihre emotionale und persönliche Entwicklung. Bedingung dafür ist eine Gesprächs- und Forschungsgemeinschaft, die sich durch einen freundlichen und wertschätzenden, respektvollen und konstruktiven Umgang der Gesprächsteilnehmer/innen auszeichnet. Obwohl die affektiven Dimensionen des Philosophierens mit Kindern wie Toleranz, Offenheit, Selbstwertschätzung und Selbstvertrauen, Kooperation und demokratische Umgangsformen im Zentrum des Ansatzes stehen, gibt es hierzu bislang verhältnismäßig wenig empirische Forschung. Das liegt unter anderem daran, dass solche vor allem qualitativ ausgerichteten Studien ungleich schwieriger und aufwändiger sind als quantitative Untersuchungen zu kognitiven Fähigkeiten auf der Basis standardisierter Testverfahren aus der Psychologie. Solche Untersuchungen sind jedoch wichtig, weil es beim Philosophieren mit Kindern als einem pädagogischen Ansatz nicht in erster Linie um eine Verbesserung der schulischen Leistungen von Kindern geht, sondern vor allem um persönlichkeitsrelevante und bildungswirksame Prozesse (vgl. Michalik 2016) und um die sozialen und politischen Konsequenzen von Erziehung und Bildung. Was Lipman als Urheber der Philosophischen Forschungsgemeinschaft im Sinne Deweys interessierte, war die Entwicklung der für die Errichtung und Erhaltung einer demokratischen Gesellschaft erforderlichen Denkfähigkeiten sowie eine allgemeine Verbesserung der Qualität von Erziehung und Bildung (vgl. Lipman/Sharp 1994).

Empirische Untersuchungen zum Philosophieren, die sich auf die soziale, emotionale und persönliche Entwicklung von Kindern und Jugendlichen richten, zeichnen sich durch eine Vielfalt verschiedenster sowohl quantitativer als auch qualitativer Forschungszugänge aus. Im Rahmen der schottischen Begleitstudie zum Schulprojekt in Clackmannanshire (siehe oben) zu den Effekten der Integration philosophischer Gespräche in das Schulcurriculum wurden neben der Entwicklung der Denkfertigkeiten der Kinder in einer Nebenstudie 
auch sozio-emotionale Effekte untersucht, weil man davon ausging, dass das Philosophieren unter Gleichaltrigen für die Entwicklung sozialer und kommunikativer Fähigkeiten, die Förderung von Empathie und Sensibilität für die Gefühle anderer als auch die Entwicklung von Selbstvertrauen und Selbstwertgefühl besonders geeignet sei (Trickey/Topping 2006: 600). In einem Pre-PostTest-und Kontrollgruppendesign mit insgesamt acht Schulklassen im Alter von 10-11 Jahren wurde auf der Basis eines standardisierten Testverfahrens Veränderungen im Selbstkonzept als Lerner und Problemlöser nach sieben Monaten Philosophieren festgestellt. Es zeigte sich eine deutliche Verbesserung des Selbstwertgefühls und Selbstvertrauens als Lerner auch im Sinne metakognitiven Denkens sowie eine Verminderung von Abhängigkeitsgefühlen und Ängsten, wobei die Effekte bei Mädchen stärker waren als bei Jungen (ebd.: 608f.). Eine begleitende Untersuchung, in der subjektive Selbstaussagen der Schüler/innen auf der Basis von Fragebögen und Interviews erhoben wurden, erweiterte und bestätigte diese Ergebnisse dahingehend, dass die Kinder mehrheitlich neben einer Verbesserung ihrer Denk- Urteils- und Konzentrationsfähigkeit von positiven Veränderungen in den Bereichen Sozialverhalten und soziale Beziehungen, Selbstbewusstsein, emotionaler Selbstkontrolle und Empathie berichteten (Trickey/Topping o.J.: 13f.). Diese Selbstaussagen konnten durch Rückmeldungen von Lehrkräften bestätigt werden (ebd.: 18f.). Die Befunde entsprechen der älteren Untersuchung von Fields, in der bei Grundschulkindern infolge regelmäßigen Philosophierens eine allgemeine Steigerung des Selbstbewusstseins und Selbstkonzepts als eigenständige Denker sowie eine Verbesserung des Sozialverhaltens erkennbar war (Fields 2006: 117f.).

Die bereits erwähnte britische Untersuchung der „Education Endowment Foundation" zu Auswirkungen des Philosophierens auf Lesefähigkeiten und mathematische Kompetenzen wurde nach Abschluss der Untersuchung durch eine weitere Studie ergänzt, die sich mit den im Rahmen der Untersuchung auch deutlich gewordenen nicht-kognitiven Effekten des Philosophierens, u.a. Steigerung des Selbstwertgefühls der Kinder, Verbesserung des Kommunikationsverhaltens, beschäftigt (Siddiqui/Gorard/See 2017). In der Folgestudie, an der 42 Schulen mit insgesamt 2722 Schüler/innen als Test- und Kontrollgruppen teilnahmen, wurde nach 18 Monaten Philosophieren in den Klassen 4-6 auf der Grundlage von Interviews mit Schüler/innen, der Bearbeitung von Textvignetten und Beobachtungen und Berichten von Lehrkräften Hinweise auf eine Verbesserung des Sozial- und Gesprächsverhaltens der Schüler/innen gefunden, die sich in einer Abnahme von Unterrichtsstörungen und anderen anti-sozialen Verhaltensweisen wie Bullying, einem respektvolleren Umgang mit anderen Meinungen, einer Verbesserung des Argumentierens, des Zuhörens und Aufeinandereingehens im Unterrichtsgespräch zeigte (ebd.: 30f.). In persönlichkeitsbildender Hinsicht wurde von den Lehrkräften eine Steigerung des Selbstvertrauens und des Vertrauens, eigene Fragen zu stellen, insbesondere bei stillen und leistungsschwachen Schüler/innen beobachtet. Auch in 
dieser Studie waren wie in den verschiedenen Studien zur kognitiven Entwicklung der Schüler/innen die von Schüler/innen selbst berichteten und von Lehrkräften beobachteten Effekte bei sozial benachteiligten Schüler/innen besonders deutlich (ebd.: 6). Von besonderem Interesse sind auch die Auswirkungen, die von den Lehrkräften, die mit den Kindern regelmäßig philosophierten, an sich selbst beobachtet wurden. Berichtet wurde u.a. von einer Veränderung des eigenen Unterrichtsstils auch in den anderen unterrichteten Fächern im Sinne von mehr Offenheit und Raum für Schüler/innenfragen und von weniger Kontrolle und mehr Autonomie und Partizipation der Schüler/innen im Sinne einer kollaborativen Lernatmosphäre (ebd.: 28).

$\mathrm{Zu}$ Veränderungen im Sozial- und Gesprächsverhalten von Kindern, die regemäßig philosophieren, gibt es weitere kleinerer Studien aus verschiedenen kulturellen Kontexten. Mit standardisierten Testverfahren konnte in Teheraner Grundschulklassen eine Verbesserung von sozialen Problemlösungsfähigkeiten festgestellt werden (vgl. Hedayati/Ghaedi 2009). In einer isländischen qualitativen Untersuchung zum Gesprächsverhalten 10-11jähriger Schüler/innen wurde eine veränderte dialogisch-demokratische Grundhaltung der Kinder beobachtet im Sinne einer Verbesserung der Interaktionen zwischen den Kindern und auch des respektvollen Umgangs mit abweichenden Meinungen (vgl. Pálsson 1996: 25ff.). Eine weitere qualitative Untersuchung aus Schweden, die Entwicklungen kommunikativer Interaktionen bei 10-jährigen Kindern in einer Test- und einer Kontrollgruppe verglich, kam zu einem vergleichbaren Ergebnis. Hier unterschieden sich die im Philosophieren geübten Kindern vor allem durch ihre besondere Toleranz und Offenheit für andere Standpunkte und Perspektiven und die Bereitschaft, eigene Meinungen zu modifizieren (vgl. Niklasson/Ohlsson/Ringborg 1996). Dass der philosophischen Gespräche kennzeichnende Umgang mit offenen und mehrdeutigen Fragen, die Entwicklung mehrperspektivischen Denkens und einen konstruktiven Umgang mit mehrdeutigen Situationen im Sinne von Ambiguitätstoleranz besonders anregt, ist auch das Ergebnis der Detailstudie von Helzel (2018). Zur Erforschung konkreter sozialer und politischer Implikationen dieser sozialen und affektiven Dimensionen des Philosophierens mit Kindern gibt es erste Ansätze. In einer Studie der Universität Quebec in Montreal haben Schleifer und Poirier (1996) auf der Basis standardisierter Tests und Interviews Wirkungen philosophischer Gespräche auf rassistische und sexuelle Stereotype von Grundschulkindern untersucht und eine deutliche Abnahme insbesondere rassistischer Stereotype festgestellt. Das Ergebnis wird von den Autorinnen dahingehend gedeutet, dass das Philosophieren die Urteilsfähigkeit der Kinder stärkt, indem es vorschnelle Schlussfolgerungen und Verallgemeinerungen vermindert und differenziertes und facettenreiches Denken, nachdenkliches Abwägen und begründetes Urteilen stärkt (vgl. hierzu auch die Ergebnisse von Tiedemann 2004). Auch die Erfahrung, dass es auf viele Fragen keine klare und sofortige Antwortmöglichkeit gibt, wird im Sinne der Entwicklung von Ambiguitätstoleranz für die 
Verminderung von Vorurteilen und Stereotypen verantwortlich gemacht (ebd.: 34), wobei der in dieser Studie erfolgte inhaltliche Fokus auf die Beschäftigung mit Gleichheit und Verschiedenheit, Augenschein und Wirklichkeit das Ergebnis mit beeinflusst haben dürfte.

$\mathrm{Ob}$ das Philosophieren eine Strategie gegen Fremdenfeindlichkeit und Rassismus sein könne, ist in einer österreichischen Studie auf der Basis qualitativer Forschungsmethoden (Analyse von Interviews, Aufsätzen von Kindern, Bildbeschreibungen) untersucht worden. Es wurden Einstellungen von Grundschulkindern gegenüber fremden Menschen erfasst, um festzustellen, ob und inwieweit das Philosophieren mit Kindern Einfluss auf diese Einstellungen nehmen kann. Das Ergebnis der Studie, dass die Versuchsklassen, in denen philosophiert wurde, am Ende des Projektes eine deutlich positivere Haltung gegenüber Fremden aufwies als die Kontrollgruppe (Camhy 2007: 138f.), wird wie in der Studie von Schleifer und Poirier auf die durch das Philosophieren gestärkte Fähigkeit zur Reflexion und zum kritischem Hinterfragen zurückgeführt (ebd.: 144), wobei jedoch auch hier die inhaltlichen Dimensionen des Projekts, das auf den Umgang mit Fremden konzentriert war, mit in Betracht zu ziehen.

\section{Wirkungen des Philosophierens auf fachliches Lernen und Unterricht - Philosophieren als Unterrichtsprinzip}

In den bisher vorgestellten Studien fand das Philosophieren mit Kindern zumeist im Rahmen eigens dafür eingerichteter Stunden, also separat vom üblichen Unterrichtsgeschehen, statt. Es gibt eine kleine Zahl von Studien, die für das Philosophieren als Unterrichtsprinzip von besonderem Interesse sind, weil hier auf der Basis überwiegend qualitativer Forschungsmethoden Wirkungen des Philosophierens im Rahmen von Fachunterricht und Auswirkungen regelmäßigen Philosophierens auf die Beschäftigung mit den Unterrichtsgegenständen untersucht worden sind. In diesen Studien wird deutlich, dass die Integration philosophischer Gespräche in den Fachunterricht sowohl die Inhalte als auch das Unterrichten und Lernen im Fach und die Interaktionsmuster im Unterricht in verschiedener Hinsicht beeinflusst.

Im Hinblick auf die Inhalte des Lernens gibt es verschiedene Hinweise darauf, dass die Integration philosophischer Gespräche in den Fachunterricht zu einer Vertiefung und Vernetzung der Unterrichtsgegenstände beiträgt und das Lernen für die Kinder subjektiv bedeutsamer macht. In den qualitativen Studien der kanadischen Forschungsgruppe um Marie-France Daniel wurden philosophische Gespräche mit Grundschulkindern in den Mathematikunterricht integriert und es wurden Fragen thematisiert, die sich auf das Wesen der Mathematik als Wissenschaft richten wie zum Beispiel: Was ist eine Zahl? 
Bedeutet Null gar nichts? Wurde Mathematik erfunden oder entdeckt? Gibt es einen perfekten Würfel? Wozu braucht man Mathematik? Ein wichtiges Ergebnis der kanadischen Studien lautet, dass das Gespräch mit den Gleichaltrigen zu einer besonderen Komplexität und Tiefe der auf den Unterrichtsgegenstand bezogenen inhaltlichen Reflexion über mathematische Probleme führte (Daniel u.a. 2005a: 249; Daniel 2005, 2013), das mathematische Verständnis der Schüler/innen verbesserte und auch Haltungen und Einstellungen der Schüler/innen zum Fach Mathematik durch philosophische Gespräche positiv beeinflusst wurden (Daniel u.a. 1999: 427).

In einer australischen Interventionsstudie, in der philosophische Gespräche in das sowohl natur- als auch sozialwissenschaftliche Inhalte umfassende Grundschul-Curriculum ,Studies of Society and Environment' integriert wurden, fanden sich ebenfalls deutliche Hinweise darauf, dass das auf die Unterrichtsgegenstände bezogene Philosophieren vor allem zu ethischen Fragen sich einerseits in einer deutlichen Verbesserung der ethischen Reflexions- und Urteilsfähigkeit auswirkte und andererseits auch in besonderem Maße zur Vertiefung und Differenzierung der inhaltsbezogenen Auseinandersetzungen führte (vgl. Collins 2005) Die Bedeutung des Philosophierens im Unterricht wird unter anderem lerntheoretisch damit begründet, dass das eigene Fragenstellen der Kinder und das gemeinsame Aushandeln von Bedeutungen wichtige Elemente für Verstehensprozesse im Unterricht sind und auch zur Lernmotivation der Schüler/innen beitragen (vgl. Knight/Collins 2012: 306f.).

Zum Philosophieren im naturwissenschaftlichen Unterricht gibt es bislang noch wenig Materialien für eine philosophische Anreicherung des Unterrichts und entsprechend gering ist die Zahl der Studien, die Wirkungen des Philosophierens auf fachliche Inhalte gewidmet sind (vgl. Sprod 2014: 1547). In einer in England durchgeführten Fallstudie mit Pre-Post, Versuchs- und Kontrollgruppendesign hat Sprod auf der Basis eines standardisierten Tests und der qualitativen Analyse von Unterrichtstranskripten untersucht, wie sich regelmäBiges Philosophieren im Biologieunterricht auf der Basis fachspezifischer Impulsmaterialien (vgl. Sprod 2011) auf die Fähigkeit von 11- bis 12-jährigen Schüler/innen zum wissenschaftlichen Argumentieren auswirkt. Nach Sprod ist der traditionelle Naturwissenschaftsunterricht nicht dazu geeignet, kritisches wissenschaftliches Denken zu fördern, weshalb es anderer pädagogischer Zugänge bedarf (Sprod 2014: 1531). Neben einer Verbesserung der Argumentationsfähigkeiten, die bei den Mädchen noch deutlicher ausfiel als bei den Jungen, zeigte sich eine Zunahme der von den Schüler/innen eingebrachten Fragen und der Interaktionen zwischen den Schüler/innen. Auf der inhaltlichen Ebene wurde deutlich, dass die Schüler/innen zunehmend in der Lage waren, Verbindungen sowohl zwischen fachlichen Inhalten und Konzepten, als auch zwischen den im Unterricht behandelten wissenschaftlichen Inhalten mit ihren eigenen lebensweltlichen Wissensbeständen und Erfahrungen herzustellen (vgl. Sprod 1997: 13f.) Eine weitere qualitative Fallstudie zum 
Biologieunterricht in einer fünften Klasse kam zu dem Ergebnis, dass die Verbindung von philosophischen Gesprächen und fachlichem Lernen dem Verstehen von Prozessen naturwissenschaftlichen Arbeitens und Denkens und der Entwicklung metakognitiven Denkens besonders förderlich ist (vgl. Ferreira 2012).

Diese in Fallstudien gewonnenen Erkenntnisse werden durch verschiedene australische Studien auf eine breitere Basis gestellt. Nichols, Burgh und Kennedy haben im Rahmen einer an neun Schulen im Test-Kontrollgruppen-Design durchgeführten zweijährigen Untersuchung mit 12- bis 13-jährigen Schüler/innen auf der Basis der Analyse von Unterrichtsgesprächen festgestellt, dass die Integration philosophischer Gespräche in einen auf forschendes Lernen ausgerichteten naturwissenschaftlichen Unterricht bei den Schüler/innen zur Entwicklung eines veränderten Frageverhaltens und eines forschenden Habitus (Fragenstellen, Ideen entwickeln, Schlüsselkonzepte untersuchen, Hypothesen testen, Schlussfolgerungen ziehen) in besonderem Maße beiträgt (Nichols/Burgh/Kennedy 2015). In dieser wie auch in einer weiteren Studie an fünf Grundschulen unter Beteiligung von 59 Grundschullehrkräften wurden ferner Hinweise darauf gefunden, dass neben dem forschenden Lernen auch das Lernen in Zusammenhängen (,connected learning“) bezogen auf das Hintergrundwissen der Schüler/innen und den Bezug zur Welt außerhalb des Klassenzimmers sowie metakognitives Denken durch das Philosophieren besonders befördert wurde (Scholl/Nichols/Burgh 2014, 2016). Ein Fazit zu den verschiedenen australischen Studien lautet, dass die Verbindung von forschendem Lernen und Philosophieren im Fachunterricht zu einer tiefgreifenden Transformation des Lernens und Denkens führt (vgl. Nichols/Burgh/Fynes-Clinton 2017: 248).

$\mathrm{Zu}$ Wirkungen des Philosophierens als Unterrichtsprinzip auf das Lernen im Fachunterricht sind ferner Forschungsbefunde aus der Biologiedidaktik relevant, in der die Bedeutung von „Alltagsphantasien“ (Gebhard 2007) für Lernprozesse von Schüler/innen untersucht worden sind. Es handelt sich hier um ein dem Philosophieren mit Kindern verwandtes Konzept, weil die auf die Unterrichtsgegenstände bezogene Assoziationen, Vorstellungen und Deutungen der Schüler/innen im Rahmen philosophischer Gespräche erhoben und bearbeitet werden. Die diesem Ansatz zugrunde liegende Annahme, dass fachliche Lernprozesse dann besonders erfolgreich sind, wenn die alltäglichen, subjektivierenden, intuitiven oder symbolischen Zugänge zu den Phänomenen im Unterricht zum Gegenstand expliziter Reflexion gemacht werden, konnte durch verschiedene Interventionsstudien empirisch fundiert werden. Es konnte gezeigt werden, dass solche Gespräche sich positiv auf die Aneignung fachwissenschaftlicher Inhalte, nachhaltiges Lernen und die Lernmotivation auswirken und dazu beitragen, das Lernen für die Schüler/innen subjektiv bedeutsamer zu machen (vgl. Combe/Gebhard 2012: 112ff.). Dass Fachunterricht von (philosophischen) Gesprächen besonders profitiert, ist auch das Ergebnis 
fachdidaktischer Forschung, die sich mit den Möglichkeiten der Förderung von Wissenschaftsverständnis im Sinne des Natur of Science-Ansatzes im naturwissenschaftlichen Unterricht beschäftigt. Auch hier konnte für den Sachunterricht der Grundschule nachgewiesen werden, dass die explizite Thematisierung erkenntnis- und wissenschaftstheoretischer Inhalte einen positiven Einfluss auf das Verstehen und Erinnern von Unterrichtsinhalten hat (vgl. Grygier u.a. 2003; Grygier 2008).

Die Befunde zur Integration des Philosophierens mit Kindern in den Fachunterricht enthalten deutliche Hinweise darauf, dass philosophisches Fragen und Forschen nicht nur die allgemeine kognitive Entwicklung der Kinder, sondern auch das inhaltsbezogene fachliche Lernen im Hinblick auf das Erreichen anspruchsvoller fachlicher Lernziele und -prozesse entschieden zu bereichern vermag. Die bisher vorgestellten Forschungsarbeiten beziehen sich ausschließlich auf fachliche Lern- und Verstehensprozesse auf Seiten der Schüler/innen. In den Studien aus Australien sind parallel hierzu auch Lernprozesse von Lehrkräften, die das Philosophieren mit Kindern im Fachunterricht eingeführt und erprobt haben, erstmals systematisch untersucht worden. Die Ergebnisse zeigen eindrucksvoll, dass das Philosophieren im Fachunterricht auch für die Lehrkräfte mit tiefgreifenden Lernprozessen verbunden ist, die zu Veränderungen im eigenen Denken und pädagogischen Handeln führen. Das Philosophieren im Unterricht führt nicht nur zu einer Intensivierung bei der Aneignung fachlicher Inhalte, sondern es verändern sich auch die Formen der Interaktionen zwischen Schüler/innen und Lehrkräften, weil Lehrer/innen und Schüler/innen gemeinsam über philosophische Fragen reflektieren und gemeinsam Dispositionen $\mathrm{zu}$ tieferem Denken und Forschen entwickeln (vgl. Scholl/Nichol/Burgh 2016: 439; Nichols/Burgh/Fynes-Clinton 2017: 245). Auf Seiten der Lehrkräfte wurde ferner eine neue Wertschätzung für die Beiträge von Schüler/innen festgestellt sowie das Infragestellen und Durchdenken des eigenen Frageverhaltens und der Gesprächsführung im Unterricht (Nichols/Burgh/Fynes-Clinton 2017: 246). Wie die Autoren unter anderem auf der Basis vergleichender Studien feststellen, ist es für einen am forschenden Lernen orientierten Unterricht nicht ausreichend, im Unterricht nur Denk- und Forschungsfertigkeiten zu schulen, sondern es kommt darauf an, bei den Schüler/innen und Lehrkräften eine Disposition zu kritischem Denken und Zweifel im Sinne einer philosophischen Grundhaltung zu entwickeln und zu kultivieren, auch im Sinne einer Beschäftigung mit eigenen Denkgewohnheiten und ontologischen Vorstellungen über die Welt (ebd.: 248f.).

In den australischen Studien sind Wirkungen des Philosophierens im Fachunterricht auf Schüler/innen und Lehrkräfte gezielt untersucht worden. $\mathrm{Zu}$ ähnlichen Ergebnissen sind Studien gelangt, die das Philosophieren im Rahmen eigener Philosophiereinheiten außerhalb des regulären Unterrichts im Hinblick auf Wirkungen im kognitiven und sprachlichen Bereich untersucht haben (vgl. zum Beispiel Trickey/Topping 2007b, 2007d; Fair 2015a: 
Siddiqui/Gorard/See 2017: 31f.). Auch hier ist im Rahmen qualitativer Begleitstudien, in denen Schüler/innen und Lehrkräfte zu ihren subjektiven Erfahrungen und Einschätzungen auf der Basis von Fragebögen und Interviews befragt wurden, deutlich geworden, dass regelmäßiges Philosophieren offenbar auf viele Bereiche des schulischen Lebens ausstrahlt und das Lernen und Lehren im Unterricht nachhaltig und positiv beeinflusst (vgl. hierzu auch den Beitrag von Michalik, Ungewissheit in diesem Band).

\section{Fazit}

Als Fazit der vorliegenden Befunde zu Wirkungen des Philosophierens mit Kindern lässt sich festhalten, dass es deutliche Hinweise darauf gibt, dass philosophische Gespräche selbst in bescheidenem Umfang, in wöchentlichen einstündigen Einheiten, im Rahmen normaler Klassenstärken und unterschiedlichen Voraussetzungen der Lehrkräfte, empirisch belegbare positive Auswirkungen hat. Das gilt sowohl im Hinblick auf kindliche Entwicklungs- und Lernprozesse als auch für die professionelle Entwicklung von Lehrkräften im Sinne einer Veränderung von Interaktionsmustern im Unterricht, die der kognitiven, sozialen und emotionalen Entwicklung der Kinder förderlich sind. Auch wenn die verschiedenen Studien nicht direkt vergleichbar sind, nicht zuletzt, weil die angewandten Methoden und Rahmenbedingungen des Philosophierens nicht einheitlich sind, weisen die Untersuchungen darauf hin, dass die Kultivierung eines auf philosophische Fragen, mehrdeutige und komplexe Sachverhalte bezogenen Unterrichtsgesprächs $\mathrm{zu}$ verbesserten kognitiven Leistungen, aber auch zu verschiedenen positiven persönlichkeitsrelevanten Entwicklungen sowohl auf Seiten der Schüler/innen als auch der Lehrkräfte führen kann.

Bei der Interpretation und theoretischen Einbettung der Ergebnisse werden die beobachteten Effekte überwiegend im Kontext sozio-konstruktivistischer Theorien der kognitiven Entwicklung und des Lernens gesehen (z.B. Vygotsky, Bruner), nach denen Kinder ihr Denken kollektiv durch Sprache in sozialen Kontexten entwickeln (vgl. z.B. Knight/Collins 2010: 316; Daniel u.a. 2005: 349; Topping/Trickey 2007b: 287). Das Philosophieren mit Kindern ist eine Methode und ein pädagogischer Ansatz, bei der sich kognitive Herausforderungen auf der inhaltlichen Ebene mit einem konsistenten Prozess des gemeinsamen Forschens, Nachdenkens und Reflektierens im Austausch mit anderen verbinden. Es handelt sich hier um einen Zugang, der Kinder auf eine besondere Art und Weise darin zu unterstützen scheint, ihre kognitiven, metakognitiven, sozialen und emotionalen Fähigkeiten und auch ein tieferes inhaltsbezogenes Verständnis zu entwickeln als im Rahmen herkömmlicher, stärker auf das individuelle Lernen der Kinder bezogenen Unterrichtsformen. 
Für zukünftige Forschung wäre eine weitere Ausdifferenzierung der Fragestellungen und Befunde interessant. $\mathrm{Zu}$ nennen sind hier Wirkungen des philosophischen Gespräches in Abhängigkeit von sozio-ökonomischen oder ethnischen Faktoren, unterschiedlichen Begabungen oder auch dem Geschlecht der Kinder sowie insgesamt eine stärkere Berücksichtigung gender- und diversitybezogener Fragestellungen (vgl. z.B. Reed-Sandoval/Sykes 2017). Damit verbunden ist die Frage nach Wirkungen philosophischer Gespräche in unterschiedlichen, zum Beispiel auch inklusiven Lernsettings sowohl im Hinblick auf das Handeln, die Haltungen und Einstellungen von Lehrkräften als auch Interaktionsprozesse in den Lerngruppen.

Die vorliegenden empirischen Befunde zu Wirkungen regelmäßigen Philosophierens sind eindrucksvoll und sprechen für eine Integration philosophischer Gespräche mit Kindern in den Unterricht. Eine allein mit den kognitiven, sozialen und anderweitigen Wirkungen begründete Einbeziehung des Philosophierens in das schulische Leben wäre jedoch einseitig und könnte auf eine Instrumentalisierung des philosophischen Gespräches hinauslaufen (vgl. Biesta 2011: 307). Das Philosophieren hat jedoch eigene spezifische Ziele, die sich nicht auf schulisches Lernen reduzieren lassen. Philosophische Gespräche tragen zur Entwicklung individueller und kollektiver Dispositionen und Fähigkeiten bei, die für die Gestaltung eines sinn- und bedeutungsvollen Lebens wichtig sind, wie zum Beispiel Freude an der intellektuellen Herausforderung und am gemeinsamen Problemlösen, persönliche Autonomieerfahrung, Verbesserung der Urteilskraft (vgl. Gregory 2013:10). Es handelt sich um eine ganzheitliche, sinnvolle Erfahrung, die zur Verbesserung der Qualität des Lebens von Kindern und Jugendlichen ganz direkt und unmittelbar beizutragen vermag.

\section{Literatur}

Biesta, Gert (2011): Philosophy, Exposure and Children: How to Resist the Instrumentalisation of Philosophy in Education. In: Journal of Philosophy of Education, 45, S. 305-319.

Camhy, Daniela G. (2007): Kann das Philosophieren eine Strategie gegen Fremdenfeindlichkeit und Rassismus sein? In: Marsal, E. et al. (Hrsg.): Ethische Reflexionskompetenz im Grundschulalter. Konzepte des Philosophierens mit Kindern. Frankfurt a. Main: Peter Lang, S. 131-144.

Cebas, Esthe/Moriyón, Felix Garcia (o. J.): What we know about Research in Philosophy for Children. https://de.scribd.com/document/231808910/What-We-KnowAbout-Research-1\#, zuletzt abgerufen am 2.10.2017

Collins, Carol (2005): Education for a just democracy: the role of ethical inquiry. Unpublished PhD Thesis, University of South Australia, Adelaide. 
Colom, Roberto/Moriyón, Félix Garcia/Magro, Carmen/Morilla, Elena (2014): The Long-term Impact of Philosophy for Children: A Longitudinal Study (Preliminary Results). In: Analytical Teaching and Philosophical Praxis, 35,1, S. 50-56.

Combe, Arno/Gebhard, Ulrich (2012): Verstehen im Unterricht. Wiesbaden: SpringerVS

Daniel, Marie-France (2005): Pour l'apprentissage d'une pensée critique au primaire. Quebec: Presses de l'Université du Quebec.

Daniel, Marie-France (2008): Learning to philosophize: Positive impacts and conditions for implementation. In: Thinking: The Journal of Philosophy for Children, 18,4 , S. 36-47.

Daniel, Marie-France (2012): Engaging in Critical Dialogue about Mathematics. In: Analytic Teaching and Philosophical Praxis, 34, 1, S. 58-68.

Daniel, Marie-France et al. (1999): Philosophical Reflection and Cooperative Practices in an Elementary School Mathematics Classroom. In: Canadian Journal of Education, 24, 4, S. 426-440.

Daniel, Marie-France et al. (2002): Are the philosophical exchanges of pupils aged 10 to 12 relativistic or inter-subjective? In: Critical and Creative Thinking, 10/2, S. 119

Daniel, Marie-France et al. (2005a): Modelling the Development Process of Dialogical Critical Thinking in Pupils Aged 10 to 12 Years. In: Communication Education, 54, 4, S. 334-354.

Daniel, Marie-France et al. (2005b): Are the philosophical exchanges of pupils aged 10 to 12 relativistic or inter-subjective? In: Critical and Creative Thinking, 10, 2, S. $1-19$.

Daniel, Marie-France/Delsol, Alain (2005): Learning to dialogue in Kindergarten. A Case Study. In: Analytic teaching, 25, 3, S. 23-52.

Daniel, Marie-France/Pettier, Jean Charles/Auriac-Slusarczyk, Emmanuele (2011): The Incidence of Philosophy on Discursive and Language Competence in FourYear-Old-Pupils. In: Creative Education, 2/3, S. 296-304.

Daniel, Marie-France et al. (2011): Developmental Process of Dialogical Critical Thinking in Groups of Pupils Ages 4 to 12 Years. In: Creative Education 2, 5, S. 418-428.

Daniel, Marie-France/Gagnon, Mathieu (2012): Pupil's Age and Philosophical Praxis: Two factors, that influence the development of critical thinking in children. In: Childhood and Philosophy 8, 15, S. 115-142.

Daniel, Marie-France/Gagnon, Mathieu/Auriac-Slusarczyk (2017): Dialogical critical thinking in kindergarten and elementary school. Studies on the impact of philosophical praxis in pupils. In: Gregory, M. R./Haynes, J./Murris, K. (Hrsg.): The Routledge International Handbook of Philosophy for Children. New York: Routledge, S. 236-244.

De Boer, Heike (2015): Lernprozesse in Unterrichtsgesprächen. In: de Boer, H./Bonanati, M. (Hrsg.): Gespräche über Lernen - Lernen im Gespräch. Wiesbaden: Springer, S. 17-36.

Fair, Frank et al. (2015a): Socrates in the Schools from Scotland to Texas: Replication a study on the effects of a Philosophy for Children program. In: Journal of Philosophy in Schools, 2, 1, S. 18-37.

Fair, Frank et al. (2015b): Socrates in the schools: Gains at three-year follow-up. In: Journal of Philosophy in Schools, 2, 2, S. 5-16. 
Fields, Joyce I. (2006): Empirical data research into the claims for using philosophy techniques with young children. In: Early Child Development and Care, 107, 1, S. 115-128.

Ferreira, Louise Brandes Moura (2012): Philosophy for children in the science class: children learning basic science process skills through narrative. Thinking: The Journal of Philosophy for Children, 20, 1, S. 71-79.

Garcia-Moriyon, Felix/Rebollo, Irene/Colom, Roberto (2004): Evaluating Philosophy for Children: A meta-analysis. In: Thinking: The Journal of Philosophy for Children, 17,4 , S. 14-22.

Gebhard, Ulrich (2007): Intuitive Vorstellungen bei Denk- und Lernprozessen: Der Ansatz der „Alltagsphantasien“. In: Krüger, D./Vogt. H. (Hrsg.): Theorien in der biologiedidaktischen Forschung, S. 117-128. Berlin: Springer

Gholamhossein, Shahini/Siamak, Samani (2010): The development of L2 speaking skills and the related components: Insight from philosophical questions. In: Procedia - Social and Behavioral Science, 5, S. 716-721.

Gorard, Stephen/Siddiqui, Nadia/See, Beng Huat (2015): Philosophy for Children. Evaluation Report and Executive Summary. School of Education. Durham University.

Gregory, Maughn Rollins (2013): Wisdom and Other Aims for Precollege Educational Objectives. In: Glina, M. B. (Hrsg.): Philosophy For, With and Of Children. Newcastle upon Tyne: Cambridge Scholars Publishing, S. 3-20.

Grygier, Patricia et al. (2004): Über Naturwissenschaften lernen. Vermittlung von Wissenschaftsverständnis in der Grundschule. Baltmannsweiler: Schneider.

Grygier, Patricia (2008): Wissenschaftsverständnis von Grundschülern im Sachunterricht. Bad Heilbrunn: Klinkhardt.

Grygier, Patricia et al. (2003): Unterstützt das Lernen über Naturwissenschaften das Lernen von naturwissenschaftlichen Inhalten im Sachunterricht? In: Cech, D./Schwier, H.-J. (Hrsg.): Lernwege und Aneignungsformen im Sachunterricht (59-76). Bad Heilbrunn: Klinkhardt.

Hedayati, Mehrnoosh/Ghaedi, Yahya (2009): Effects of the philosophy for children program through the community of inquiry method on the improvement of interpersonal relationship skills in primary school students. In: Childhood and Philosophy, 5, 9, S. 201-217.

Hausberg, Anna K. (2013): Fressen Katzen Rotklee? Kreativität beim Philosophieren mit Kindern und Jugendlichen. Marburg: Tectum.

Helzel, Gudrun (2018): Kindliche Entwicklungsprozesse beim Philosophieren mit Kindern. Eine empirische Studie zu Ungewissheit und Mehr-Perspektivität. Opladen, Berlin, Toronto: Barbara Budrich.

Helzel, Gudrun/Michalik, Kerstin (2016): Kindliche Entwicklungsprozesse beim Philosophieren mit Kindern. Eine empirische Untersuchung zu Mehr-Perspektivität und Ungewissheitstoleranz. In: Fischer, H.J./Giest, H./Michalik, K. (Hrsg.): Bildung im und durch Sachunterricht. Bad Heilbrunn: Klinkhard, S. 189-196.

Knight, Sue/Collins, Carol (2010): Enlivening the curriculum: The power of philosophical inquiry. In: Theory and Research in Education 8, 3, S. 305-318.

Lafortune, Louise et al. (2000). Evolution of Pupil's Attitudes to Mathematics When Using a Philosophical Approach. In: Analytic Teaching, 20/1, S. 46-63.

Lipman, Matthew/Sharp, Ann Margaret (1994): Growing up with Philosophy. Dubuque/Iowa: Kendall/Hunt. 
Marsal, Eva/Dobashi, Takara (2009): Empirical Evaluation of Philosophy Instruction (P4C): Models, Methods, Examples. In: Marsal, E./Dobashi, T./Weber, B. (Hrsg.): Children Philosophize Worldwide. Theoretical and Practical Concepts, S: 473487. Frankfurt/M. u.a: Peter Lang.

Michalik, Kerstin (2016): Philosophische Gespräche mit Kindern als Medium für Bildungsprozesse im Sachunterricht. In: Fischer, Hans-Joachim/Giest, Hartmut/Michalik, Kerstin (Hrsg.): Bildung im und durch Sachunterricht. Bad Heilbrunn: Klinkhard, S. 175-182.

Michalik, Kerstin (2013): Philosophieren mit Kindern als Unterrichtsprinzip - Bildungs- und lerntheoretische Begründungen und empirische Fundierungen. In: Pädagogische Rundschau, 6, S. 635-649.

Niklasson, John/Ohlsson, Ragnar/Ringborg, Monika (1996): Evaluating Philosophy for Children. In: Thinking: The Journal of Philosophy for Children, 12, 4, S. 17-21.

Nichols, Kim/Burgh, Gilbert/Fynes-Clinton, Liz (2017): Reconstruction of thinking across the curriculum through the community of inquiry. In: Gregory, M.R./Haynes, J./Murris, K. (Hrsg.): The Routledge International Handbook of Philosophy for Children. London, New York: Routledge, S. 245-252.

Nichols, Kim/Burgh, Gilbert/Kennedy, Callie (2015): Comparing Two Inquiry Professional Development Interventions in Science on Primary Student's Questioning and Other Inquiry Behaviours. In: Research in Science Education, 47, 1, S. 1-24.

Pálsson, Hreinn (1996): We Think More than Before about Others and their Opinions. An Evaluation Report from Iceland. In: Thinking and Reasoning 12, S. 24-28.

Reed-Sandoval, Amy/Sykes, Alain Carmen (2017): Who talks? Who listens? In: Gregory, M. R./Haynes, J./Murris, K. (Hrsg.): The Routledge International Handbook of Philosophy for Children. London, New York: Routledge, S. 219-226.

Rohbeck, Johannes et al. (Hrsg.) (2009): Empirische Unterrichtsforschung und Philosophiedidaktik. Dresden: Thelem.

Sasseville, Michel (1995): Self-esteem, logical skills and philosophy for children. In: Thinking. The Journal of Philosophy for Children, 4, 2, 30-32.

Schleifer Michael/Courtemanche, Louise (1996): The Effect of Philosophy for Children on Language Ability. In: Thinking: The Journal of Philosophy for Children, 12, 4, S. 30-31.

Schleifer, Michael/Poirier/Ginette (1996): The Effect of Philosophical Discussions in the Classroom on Respect for Others and non-Stereotypic Attitudes. In: Thinking: The Journal of Philosophy for Children, 12, 4, S. 32-34.

Schleifer, Michael/Daniel, Marie-France/Peyronnet, Emmanuelle/Lecomte, Sarah (2003): The Impact of Philosophical Discussions on Moral Autonomy, Judgement, Empathy and the Recognition of Emotions in Five Year Olds. In: Thinking: The Journal of Philosophy for Children, 16, 4, S. 4-12.

Scholl, Rosie/Nichols, Kim/Burgh, Gilbert (2014): Transforming pedagogy through philosophical inquiry. International Journal of Pedagogies and Learning, 9, 3, 253 272

Scholl, Rosie/Nichols, Kim/Burgh, Gilbert (2016): Connecting learning to the world beyond the classroom through collaborative philosophical inquiry. In: Asia-Pacific Journal of Teacher Education, 44, 5, S. 436-454.

Siddiqui, Nadia/Gorard, Stephen/See, Beng Huat (2017): Non-cognitive impacts of Philosophy for Children. School of Education Durham University. 
Simon, Charlann (1979): Philosophy for students with learning disabilities. In: Thinking: The Journal of Philosophy for Children 1, S. 21-34.

Sprod, Tim (1997): Improving Scientific Reasoning through Philosophy for Children: an Empirical Study. In: Thinking: The Journal of Philosophy for Children, 13/4, S. 311-316.

Sprod, Tim (2011): Discussions in Science. Promoting conceptual understanding in the middle school years. Camberwell: Acer Press.

Sprod, Tim (2014): Philosphical Inquiry and Critical Thinking in Primary and Secondary Science Education. In: Matthews, Michael R. (Hrsg.): International Handbook of Research in History, Philosophy and Science Teaching. Bd. 2, S. 1531-1564.

Sternberg, Robert J./Bhana, Kastoor (1996): Synthesis of Research on the Effectiveness of Intellectual Skills Programs: Snake-Oil remedies or Miracle Cures? In: Educational Leadership 44, 2, S. 60-67.

Tiedemann, Markus (2004): Ethische Orientierung für Jugendliche. Münster: LIT.

Tiedemann, Markus (2011): Philosophiedidaktik und empirische Bildungsforschung. Möglichkeiten und Grenzen. Berlin: LIT.

Topping, Keith J./Trickey, Steve (2007a): Collaborative philosophical enquiry for school children: Cognitive effects at 10-12 years. In: British Journal of Educational Psychology, 77, S. 271-288.

Topping, Keith J./Trickey, Steve (2007b). Collaborative philosophical enquiry for school children: Impact of philosophical inquiry on school student's interactive behaviour. In: Thinking Skills and Creativity, 2, S. 73-84.

Topping, Keith J./Trickey, Steve (2007c). Collaborative philosophical inquiry for school children: Cognitive gains at a 2-year follow-up. In: British Journal of Educational Psychology, 77, S. 787-796.

Topping, Keith J./Trickey, Steve (2007d): Collaborative Philosophical Enquiry for School children: Participant Evaluation at 11 Years, In: Thinking. The Journal of Philosophy for Children 18/3, S. 23-34.

Topping, Keith J./Trickey (2007e): Impact of philosophical enquiry on school students' interactive behaviour. In: Thinking Skills and Creativity, 2, S. 73-84.

Topping, Keith J./Trickey, Steve (2015): Philosophy for Children. Short and Long-term effects. In: Wegerif, Rupert et al. (Hrsg.): The Routledge International Handbook of Research in teaching thinking, S. 103-112.

Trickey, Steve/Topping, Keith J. (2004): „Philosphy for Children“: a systematic review. In: Research Papers in Education, 19/3, S. 365-380.

Trickey, Steve/Topping, Keith J. (2006): Collaborative Philosophical Enquiry for School Children: Socio-Emotional Effects at 11 to 12 Years. In: School Psychology International, 27, S. 599-614. 